Acta Materialia, 64 (2014) 345-355.

\title{
Origin of $\{332\}$ twinning in metastable $\beta$-Ti alloys
}

Hirobumi Tobe ${ }^{\text {a }}$, Hee Young Kim ${ }^{\mathrm{a}, *}$, Tomonari Inamura ${ }^{\mathrm{b}}$, Hideki Hosoda ${ }^{\mathrm{b}}$, Shuichi Miyazaki $\mathrm{a}, \mathrm{c}, *$

${ }^{a}$ Division of Materials Science, University of Tsukuba, Tsukuba, Ibaraki 305-8573, Japan

${ }^{\mathrm{b}}$ Precision and Intelligence Laboratory, Tokyo Institute of Technology, Yokohama 226-8503, Japan

${ }^{\mathrm{c}}$ Center of Excellence for Advanced Materials Research, King Abdulaziz University, PO Box 80203, Jeddah 21589, Saudi Arabia

*Corresponding authors. Address: Division of Materials Science, University of Tsukuba, Tsukuba, Ibaraki 305-8573, Japan. Tel./fax: +81 298536942 (H.Y. Kim), +81 298535283 (S. Miyazaki).

E-mail addresses: heeykim@ims.tsukuba.ac.jp (H.Y. Kim), miyazaki@ims.tsukuba.ac.jp (S. Miyazaki).

\begin{abstract}
In this paper, the origin of $\{332\}<11 \overline{3}>$ twinning, which is a unique twinning mode in metastable $\beta$-Ti alloys, was investigated. The possible twinning modes in metastable $\beta$-Ti alloys are derived by considering lattice instability in addition to the theory of the crystallography of deformation twinning. In order to consider lattice instability in the bcc structure, a modulated
\end{abstract}


structure was proposed. We then clarify how the modulated structure facilitates the $\{332\}<11 \overline{3}>$ twinning preferentially compared to other possible twinning modes by evaluating the magnitude of twinning shear and the complexity of shuffle. It was found that the lattice instability makes the $\{112\}<1 \overline{1} 1>$ twinning, which is a major twinning mode observed in alloys with stable bcc structure, difficult to occur because the lattice modulation causes additional shuffling to form the $\{112\}<\overline{1} \overline{1} 1>$ twin. It was also found that the $\{332\}<11 \overline{3}>$ twinning is the most possible twinning mode in the modulated structure in terms of the magnitude of twinning shear and the complexity of shuffle. The new formation model of the $\{332\}<11 \overline{3}>$ twin presented in this study can explain how the lattice instability preferentially activates the $\{332\}<11 \overline{3}>$ twinning in metastable $\beta$-Ti alloys.

Keywords: $\beta$-titanium; Deformation twin; $\{332\}$ twin; Phase stability; Martensitic transformation

\section{Introduction}

Twinning is an important deformation mechanism not only in materials with low crystal symmetry such as hep metals but also in bcc metals and fcc metals with low stacking fault energies [1]. It has been reported that deformation twinning simultaneously improves the strength and ductility of nanocrystalline materials [2] and also plays a very important role in TWIP (twinning-induced plasticity) steels as it results in an extra strain hardening [3].

Recently, $\beta$-type Ti alloys have attracted considerable attention as promising materials 
for biomedical applications due to their excellent properties such as low Young's modulus, good biocompatibility, high resistance to corrosion, shape memory effect and superelasticity [4-8]. However, the strength of $\beta$-Ti alloys is relatively lower than that of other metallic materials such as Ti-6Al-4V, stainless steel or Co-Cr-Mo alloys, which are currently being used for biomedical applications. There have been extensive efforts to increase the tensile strength of $\beta$-Ti alloys [9-11]. However, most of the cases the increase of tensile strength resulted in decrease of ductility. Min et al. [12,13] reported in Ti-Mo-based alloys that the combination of two deformation modes, i.e., twinning and slip, can enhance the uniform elongation with maintaining high strength. It has been reported that the deformation by $\{332\}<11 \overline{3}>$ twinning in $\beta$-Ti alloys caused a large elongation through the significant work hardening due to the dynamic grain refinement [14-16]. Sun et al. [17] also reported that the combined effects of phase transformation and mechanical twinning, $\{332\}<11 \overline{3}>$ and $\{112\}<\overline{1} \overline{1} 1>$, resulted in a high work hardening in a metastable $\beta$ Ti-12 wt.\% Mo alloy. It has also been pointed out that the $\{332\}<11 \overline{3}>$ twinning is closely related with the stress-induced $\omega$ phase transformation $[15,18-20]$. It is well known that the $\omega$ phase significantly affects mechanical properties such as Young's modulus, yield strength and ductility of $\beta$-Ti alloys.

In the last decade, deformation mechanisms of $\beta$-Ti alloys have attracted a great deal of interest especially in Gum Metals [21]. A dislocation-free deformation mechanism related with the formation of giant faults and the existence of nanodisturbances has been reported in Gum Metals [21-25]. An unusual twinning mode, $\{332\}<11 \overline{3}>$ twinning, is one of unique deformation mechanisms and has been reported in alloys where the amount of $\beta$ phase stabilizing alloy elements such as $\mathrm{Nb}$ and Ta is reduced from that of Gum Metals [26-29]. The decrease of the $\beta$ phase stabilizing alloy elements causes the decrease in the shear modulus $c^{\prime}$ $\left(\left(c_{11}-c_{12}\right) / 2\right)$ which reflects the resistance to shear of $\{011\}_{\beta}$ planes along $\langle 011\rangle_{\beta}$ directions. 
This strongly suggests that the lattice instability of $\beta$ phase activates the $\{332\}<11 \overline{3}>$ twinning. Up to now, the $\{332\}<11 \overline{3}>$ twinning has been observed in several metastable $\beta$-Ti alloys, such as Ti-Mo-based alloys [30-34], Ti-V-based alloys [19,35,36], Ti-Nb-based alloys [37-39] and binary Ti-Cr [40,41] and Ti-Fe [42] alloys, and considered as an important deformation mode in addition to the stress-induced martensitic transformation [17].

As described above, the $\{332\}<11 \overline{3}>$ twinning has attracted much interest as an important deformation mechanism which strongly influences the mechanical properties of $\beta$-Ti alloys. However, interestingly, the $\{332\}<11 \overline{3}>$ twinning has not been reported to occur as a major twinning mode in other bcc metals and alloys. This is because the $\{332\}<11 \overline{3}>$ twinning is not the most favorable twinning mode in bcc metals and alloys because one-half of atoms must shuffle after the twinning shear to form the $\{332\}<11 \overline{3}>$ twin [43]. Such additional movement of atoms is not required in $\{112\}<1 \overline{1} 1>$ twinning which is a well-known twinning mode formed in bcc metals and alloys. It was suggested by Hanada et al. $[18,37]$ that the stability of $\beta$ phase plays a key role in the formation of the $\{332\}<11 \overline{3}>$ twinning; however the origin of the $\{332\}<11 \overline{3}>$ twinning remains unclear.

Several models of the movement of atoms required in the $\{332\}<11 \overline{3}>$ twinning have been presented so far $[38,44-48]$, and it has been considered that lattice instability of the metastable $\beta$ phase allows such complex movement of atoms and makes the $\{332\}<11 \overline{3}\rangle$ twinning possible $[18,46,49]$. We point out however that if such a large magnitude of shuffle required in the $\{332\}<11 \overline{3}>$ twinning is allowed to occur in practice, there are many other twinning modes which are considered to be possible in addition to the $\{332\}<11 \overline{3}>$ twinning. Therefore in order to clarify a formation model of the $\{332\}<11 \overline{3}>$ twin, it is required to consider how the lattice instability preferentially activates the $\{332\}<11 \overline{3}>$ twinning. However, such possibility of other twinning modes and the comparison of the possibility of the 
$\{332\}<11 \overline{3}>$ twinning and other twinning modes have not been argued before. In this paper, we first derived the possible twinning modes in metastable $\beta$-Ti alloys by considering lattice instability in addition to the theory of the crystallography of deformation twinning proposed by Bilby and Crocker [50]. In order to consider lattice instability in the bcc structure which exhibits the martensitic transformation, we propose a double lattice structure model. We then clarify how the lattice instability facilitates the $\{332\}<11 \overline{3}>$ twinning preferentially compared to other possible twinning modes.

\section{Shuffle mechanisms of single and double lattice structures}

In order to consider the possibility of the $\{332\}<11 \overline{3}>$ twinning, it is important to pay attention to atomic shuffles which are closely related to the complexity of the twinning. The theory concerning the shuffle mechanism which is based on the deformation twinning theory of Bilby and Crocker $[50,51]$ is summarized in this section. Firstly, the shuffles involved in the twinning modes of single lattice structures are described below. The single lattice structures are built up of atoms placed at the lattice points of a single Bravais space lattice. The complexity of the shuffle mechanism is related to the $q$ value. In the case of type I twinning, $q$ corresponds to the number of lattice planes parallel to the twinning plane $\left(K_{1}\right)$; the lattice planes are intersected by a primitive lattice vector in the conjugate twinning direction $\left(\eta_{2}\right)$. Similarly, in type II twinning, $q$ is the number of planes parallel to the conjugate twinning plane $\left(K_{2}\right)$; the planes are intersected by a primitive lattice vector in the twinning direction $\left(\eta_{1}\right)$. In the case of compound twinning modes, both type I and type II orientation relationships can be considered and $q$ is calculated from $K_{1}$ and $\eta_{2}$ for the type I orientation relationship and from $K_{2}$ and $\eta_{1}$ for the type 
II orientation relationship. If the plane of shear of compound twinning modes is a symmetry plane of the lattice, the type I and type II orientation relationships are identical. Fig. 1 shows the schematic diagram of the twinning in single lattice structures for the case of $q=1,2,3$ or 4 . If $q$ $=1$ or 2 , every point of the Bravais lattice is sheared to its correct twin position as shown in Fig. 1(a) and (b). On the other hand, some of the lattice points must shuffle if $q>2$ : when $q$ is odd a fraction $q^{-1}$ of the lattice points is sheared correctly, whereas a fraction $2 q^{-1}$ is sheared correctly when $q$ is even. For example, as shown in Fig. 1(c) and (d), two-thirds of the atoms must shuffle when $q=3$ while one-half of the atoms must shuffle when $q=4$. This means that the movement of atoms for $q=3$ is more complex than that for $q=4$.

The double lattice structures have atoms at the lattice points of two interpenetrating lattices, or equivalently consist of motif units of two atoms at each lattice point of a single lattice. Twinning in the double lattice structures requires the shuffles which correspond to the rearrangement or disruption of the motif units, in addition to the shear of the lattice points. Fig. 2 and 3 illustrate the shuffle mechanisms suggested by Crocker [51] which may occur in practice for $q=1$ and $q=4$, respectively. In general, the atoms comprising a motif unit do not lie in the plane of shear, so the atoms represented by open and closed circles lie above and below the plane of shear, respectively. The shuffles required in $q=1$ modes correspond to the rearrangement of the motif units at the lattice points as shown in Fig. 2. Fig. 2(a) represents the shear of the lattice points. The motif units of two atoms at the lattice points are considered to shear as rigid bodies. After shearing, the rearrangement of the atoms in each motif unit must take place by means of mechanism Ia or Ib to produce the type I twin (Fig. 2(b)) or by means of mechanism IIa or IIb to give the type II twin (Fig. 2(c)). The shuffles involved in $q=2$ modes are the same as those in the $q=1$ modes because every lattice point is sheared to its correct twin position when $q=1$ or 2. On the other hand, in $q=4$ modes shown in Fig. 3, the rearrangement 
of the motif units takes place at only one-half of the lattice points which are sheared to their correct twin positions. The motif units at the other one-half of the lattice points are required to disrupt and form new units of two atoms by means of mechanism Ic or Id and IIc or IId to produce the type I twin (Fig. 3(b) and (c)) and type II twin (Fig. 3(d) and (e)), respectively. The diagrams of Fig. 2 and 3 illustrate different ways of the rearrangement or disruption of the motif units of two atoms for type I orientation relationship (Ia-Id) and type II orientation relationship (IIa-IId).

\section{Possible twinning modes in the bcc structure}

Firstly, the possible twinning modes in the bcc structure are considered in this section without concerning the effect of lattice instability. It is generally acknowledged that the magnitude of twinning shear and complexity of shuffle are two important factors in determining the deformation twinning modes [1]. The twinning modes with $q=1$ or 2 require no shuffling in single lattice structures. Among the $q>2$ modes in which shuffles of atoms are necessary, $q=4$ modes have the simplest shuffle mechanisms as mentioned in the previous section. Therefore, the possible twinning modes with $q=1,2$ or 4 in the bcc structure were calculated using the theory of deformation twinning. The calculated twining modes which have twinning shear $s<$ 0.8 are listed in Table 1 in order of increasing the $s$ value. All the twinning modes are compound twinning modes, and the $q$ value of each mode corresponds to the smaller one of either type I or type II orientation relationships. There are no $q=1$ modes in Table 1 because they have large shear magnitudes $s>0.8$.

In Table 1, modes 2, 4 and 7 are conjugates of modes 1,3 and 6, respectively. The 
$\{112\}<\overline{1} \overline{1} 1>$ twinning (mode 5) is a well-known twinning mode formed in bcc metals and alloys. This twinning mode is the only mode which has $q=2$ and therefore no shuffling is necessary; this explains why the $\{112\}<\overline{1} \overline{1} 1>$ twinning is the most preferential twinning mode in bcc metals and alloys. Mode 2 is the $\{332\}<11 \overline{3}>$ twinning which has been observed in metastable $\beta$-Ti alloys. The $K_{1}$ plane of modes 3 and 6 is the $\{110\}$ plane which is a plane of symmetry of the bcc structure. Such planes are not normally recognized as possible twinning planes because the twin product has the same orientation as that of the matrix. Among the $q=4$ modes, modes 1 and 2 have the smallest shear $s=1 /(2 \sqrt{2})(=0.3536)$ which is one-half of the shear of the $\{112\}<\overline{1} \overline{1} 1>$ twinning (mode 5). Fig. 4 shows the shear and the possible shuffle mechanisms of modes 1 and 2, i.e., $\{112\}<11 \overline{1}>$ and $\{332\}<11 \overline{3}>$ twinning. The red and green arrows indicate the shear and shuffle, respectively. The shuffle mechanism with the smallest magnitude of shuffle was chosen as the most possible shuffle mechanism because we consider that atoms should move to stable lattice sites with the shortest distance after the shear. The $\{112\}<11 \overline{1}>$ and $\{332\}<11 \overline{3}>$ twinning require the same amount of the magnitude of shuffle $\Delta$ $=(\sqrt{3} / 4) a_{0}\left(=0.4330 a_{0}\right)$, where $a_{0}$ is the lattice parameter of the bcc lattice. The magnitude of shuffle is denoted as $\Delta$ in this paper. It is noted that the $\{112\}<11 \overline{1}>$ and $\{332\}<11 \overline{3}>$ twinning are not observed in metals and alloys with the stable bcc structure although their smaller magnitude of shear, implying that the magnitude of shuffle $\left(\Delta=0.4330 a_{0}\right)$ is so large that the shuffles are unable to occur in practice. In metastable $\beta$-Ti alloys, it has been considered that lattice instability makes the $\{332\}<11 \overline{3}>$ twinning possible $[18,46,49]$. Considering the magnitude of shuffle, it seems possible for the $\{112\}<11 \overline{1}>$ twinning to occur in metastable $\beta$-Ti alloys in addition to the $\{332\}<11 \overline{3}>$ twinning. However the $\{112\}<11 \overline{1}>$ twinning has not been reported so far. This result indicates that lattice instability preferentially activates the $\{332\}<11 \overline{3}>$ twinning compared to the $\{112\}<11 \overline{1}>$ twinning. This should be taken into 
account when we consider the formation mechanism of the $\{332\}<11 \overline{3}>$ twin.

\section{New formation model of the $\{332\}<11 \overline{3}>$ twin}

\subsection{Lattice modulation and the possible twinning modes in the metastable $\beta$ phase}

Recently, it has been shown that a lattice modulation exists in metastable Ti-Nb-based alloys, which is due to the low shear modulus $c^{\prime}\left(\left(c_{11}-c_{12}\right) / 2\right)$ of $\beta$ phase [52-54]. The low shear modulus $c^{\prime}$ causes the shuffling of parallel adjacent $\{0 \overline{1} 1\}_{\beta}$ planes to $\langle 011\rangle_{\beta}$ directions since the shear modulus $c^{\prime}$ reflects the resistance to shear of $\{0 \overline{1} 1\}_{\beta}$ planes along $\langle 011\rangle_{\beta}$ directions. Nii et al. [52] reported the existence of diffused superlattice spots in the electron diffraction pattern taken at room temperature from the metastable $\beta$ phase of a Ti-26 at.\% $\mathrm{Nb}$ alloy (martensitic transformation start temperature, $M_{\mathrm{s}} \sim 280 \mathrm{~K}$ ). They claimed that such diffused spots are originated from the lattice modulation which corresponds to the shuffling of parallel adjacent $\{011\}_{\beta}$ planes to $\langle 011\rangle_{\beta}$ directions. However there is almost no contrast in the dark-field micrograph obtained using the superlattice spot. It is considered from the result that there is a fluctuation between the bcc structure and the modulated bcc structure in the structure of the metastable $\beta$ phase, although the average structure is bcc. We propose that the lattice modulation, i.e. the shuffling of parallel adjacent $\{0 \overline{1} 1\}_{\beta}$ planes to $\langle 011\rangle_{\beta}$ directions, plays a critical role in the formation of the $\{332\}_{\beta}<11 \overline{3}>_{\beta}$ twin.

Fig. 5(a) and (b) show the bcc structure and the modulated bcc structure, respectively. The shift of the atoms in the modulated structure indicated by the orange arrows is represented by $\delta<011>_{\beta}$, where $\delta$ is introduced to describe the amplitude of the modulation. It is considered 
that the fluctuation occurs in the condition that the amplitude of the modulation is smaller than that in the martensite structure. The structure of the $\alpha^{\prime}$ martensite phase is hcp while that of the $\alpha^{\prime \prime}$ martensite phase is orthorhombic [8,55]. If the amplitude of the modulation in the $\alpha^{\prime}$ martensite and that in the $\alpha^{\prime \prime}$ martensite are represented as $\delta_{\alpha^{\prime}}$ and $\delta_{\alpha^{\prime \prime}}$, respectively, $\delta_{\alpha^{\prime}}$ is $1 / 6$ while $\delta_{\alpha^{\prime \prime}}$ is smaller than $\delta_{\alpha^{\prime}}$ and depends on the alloy composition [56-58]. Therefore, $\delta$ is restricted to be $0<\delta<\delta_{\alpha^{\prime \prime}}<1 / 6\left(\delta_{\alpha^{\prime}}\right)$. The modulated structure can be treated as the double lattice structure consisting of atoms at the nodes of two interpenetrating base-centered tetragonal lattices as illustrated in Fig. 5(c). In order to calculate possible twinning modes in the double lattice structure, the structure is considered as the tetragonal structure shown in Fig. 5(d) which consists of motif units of two atoms at each lattice point of a single base-centered tetragonal lattice. The crystal axes in the tetragonal lattice were set in a way that the $[001]_{\mathrm{t}}$ direction is parallel to the directions perpendicular to the shuffling planes $\{0 \overline{1} 1\}_{\mathrm{c}}$ and the $[010]_{\mathrm{t}}$ direction is parallel to the shuffling directions $\langle 011\rangle_{\mathrm{c}}$, where subscripts $\mathrm{t}$ and $\mathrm{c}$ indicate base-centered tetragonal and body-centered cubic, respectively. There are six lattice correspondences between the tetragonal and bcc structures as listed in Table 2. In order to consider the twinning modes in the tetragonal structure with simple shuffle mechanisms, the possible twinning modes which have $q=1,2$ or 4 were calculated using the theory of deformation twinning, and those with $s<$ 0.8 are listed in Table 3. Considering the lattice correspondence between the tetragonal and bcc structures shown in Table 2, the predicted twinning modes in the tetragonal structure are written in the cubic Miller indices as shown in Table 3. For example, if the $\{130\}_{t}<\overline{3} 10>_{t}$ twinning (mode 6) occurs in the tetragonal structure, the twinning is observed as the $\{332\}_{\mathrm{c}}\langle 11 \overline{3}\rangle_{\mathrm{c}}$ twinning in $\beta$ phase. It is noted that all the possible twinning modes of the bcc structure shown in Table 1 can be seen in Table 3. Besides, all the possible twinning modes for $q=8$ of the bcc structure, which were not considered in the previous section, are also included in Table 3. This 
means that only the twinning modes which are possible in the bcc structure can be observed after twinning in the tetragonal structure. In the next section, we discuss the most possible twinning mode in the tetragonal structure, i.e., the most possible twinning mode in the modulated bcc structure.

\subsection{Shuffle mechanisms of the possible twinning modes in the modulated bcc structure}

In contrast to single lattice structures, atomic shuffles are necessary for twinning in double lattice structures even if the twinning modes have $q=1$ or 2 . Therefore, the complexity of the atomic shuffles is closely related to the possibility of the twinning modes. In Table 3 , the $\{110\}_{\mathrm{t}}\langle 1 \overline{1} 0\rangle_{\mathrm{t}}$ twinning of mode 29 is the only $q=1$ mode, which corresponds to the $\left.\{112\}_{\mathrm{c}}<\overline{1} 11\right\rangle_{\mathrm{c}}$ twinning. It is important to note that the $\{110\}_{\mathrm{t}}\langle 1 \overline{1} 0\rangle_{\mathrm{t}}$ twinning requires the shuffles corresponding to the rearrangement of motif units although the $\{112\}_{\mathrm{c}}\langle\overline{1} \overline{1} 1\rangle_{\mathrm{c}}$ twinning does not need any shuffles, implying that the lattice modulation makes the $\{112\}_{\mathrm{c}}\langle\overline{1} \overline{1} 1\rangle_{\mathrm{c}}$ twinning difficult to occur. There are two twinning modes $\{110\}_{\mathrm{t}}\langle\overline{1} 10\rangle_{\mathrm{t}}$ and $\{130\}_{\mathrm{t}}\langle\overline{3} 10\rangle_{\mathrm{t}}$ twinning (modes 5 and 6) which have the smallest shear for $q=2$. These $q=2$ modes require simple shuffle mechanisms of only the rearrangement of motif units similar to the $\{110\}_{\mathrm{t}}\left\langle 1 \overline{1}_{0}\right\rangle_{\mathrm{t}}$ twinning with $q=1$. The $\{110\}_{\mathrm{t}}<\overline{1} 10>_{\mathrm{t}}$ and $\{130\}_{\mathrm{t}}<\overline{3} 10>_{\mathrm{t}}$ twinning correspond to the $\left.\{112\}_{\mathrm{c}}<11 \overline{1}\right\rangle_{\mathrm{c}}$ and $\{332\}_{\mathrm{c}}<11 \overline{3}>_{\mathrm{c}}$ twinning of $q=4$, respectively. Modes $1,2,3$ and 4 with $q=$ 4 have smaller magnitudes of shear compared to modes 5 and 6 , although the shuffle mechanisms are more complex. Among the four $q=4$ modes, the $\{110\}_{t}\langle 1 \overline{1} 0\rangle_{\mathrm{t}}$ and $\{350\}_{\mathrm{t}}\left\langle 5 \overline{3} 0>_{\mathrm{t}}\right.$ twinning (modes 1 and 2$)$ have the smallest shear $s=1 /(4 \sqrt{2})(=0.1768)$, which is one-half of the shear of modes 5 and 6 . The twinning shear of modes 3 and 4 is slightly smaller than that of modes 5 and 6 . The twinning plane $\left(K_{1}\right)$ of the mode 3 is $(001)_{\mathrm{t}}$ which is a 
plane of symmetry of the tetragonal structure; therefore the mode 3 is unlikely to occur in practice because the twin product has the same orientation as that of the matrix. In the mode 4 ,

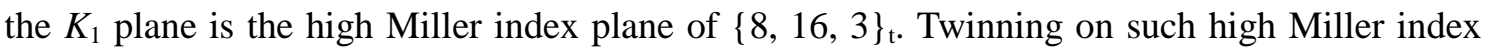
planes is not considered to be reasonable because higher Miller index planes have smaller interplanar spacing. As a result, the modes 1, 2, 5 and 6 can be considered as the strong candidates for the preferentially operative twinning modes in the tetragonal structure. We examine in detail the shuffles of the above twinning modes $1,2,5$ and 6 to discuss their possibility.

When examining shuffles in practice, the choice of the motif unit is important. There are only six crystallographically distinct pairs of atoms comprising the motif unit in the tetragonal structure, namely $\kappa, \lambda, \mu, v, o$ and $\xi$, similar to the motif units presented in $\alpha$-uranium [51]. The projections of the motif units on the (001)t plane are shown in Fig. 6(a). The vector joining the two atoms comprising a motif unit is represented by $[x y z]_{t}$, and the motif units are listed in Table 4 in order of increasing the magnitude of the units. The units $\kappa$ and $\xi$ have two crystallographically equivalent variants and the units $\lambda, \mu, o$ and $v$ have four each, thus twenty possible units arise in all. The different variants are distinguished by subscripts. The $(001)_{t}$ plane is the plane of shear of the twinning modes $1,2,5$ and 6 . The possible twinning plane position and the possible motif unit of the twinning modes are shown in Fig. 6(b)-(d). The gray dotted lines represent the planes parallel to the twinning plane in the two interpenetrating lattices. We set the twinning plane in the middle of the two planes which have smaller interplanar spacing. The smallest motif unit lying approximately parallel to the twinning plane was chosen as the possible motif unit in all cases so that the motif unit can be considered to shear as a rigid body. The magnitudes and directions of the possible shuffles associated with the twinning modes 1, 2, 5 and 6 were examined in detail. The magnitude of shuffle depends on the 
$\delta$ value, where the range of $\delta$ is restricted to be $0<\delta<1 / 6$ as mentioned in the previous section. The shuffle mechanisms which have the smallest magnitude of shuffle in the whole range of $0<$ $\delta<1 / 6$ were chosen as the most satisfactory shuffle mechanisms, and summarized in Table 5 . Figure 7 shows the magnitude of shuffle plotted in the range of $0<\delta<1 / 6$. The details of the calculation procedure of the magnitude of shuffle are described in Ref. [51]. In Table 5, type I and type II orientation relationships are given for each mode since both the orientation relationships have the same $q$ value. Mode 1 and 2 involve two shuffle mechanisms for each orientation relationship: one corresponds to the rearrangement of the motif units and the other corresponds to the disruption of them. In order to calculate the magnitudes of shuffles Ic and Id, a lattice vector lying in the twinning plane has to be chosen which defines the relative positions of the two parent motif units which disrupt to produce a single motif unit of the twin. We set the vector equal to $[\overline{1 / 2}, 1 / 2,0]_{\mathrm{t}}$ for Id of mode 1 and $[\overline{5 / 2}, 3 / 2,0]_{\mathrm{t}}$ for Ic of mode 2 so that the magnitude of shuffle has the smallest value. In modes 5 and 6 , the disruption of the motif units is not required since the modes have $q=2$.

As we can see in Fig. 7, the shuffling of the $\{110\}_{t}\langle 1 \overline{1} 0\rangle_{t}$ twinning is expected to occur for the type II orientation relationship, if possible, due to the smaller magnitude of shuffle compared to the type I orientation relationship. The magnitude of shuffle for the mechanism IIb in the $\{110\}_{t}\langle 1 \overline{1} 0\rangle_{t}$ twinning is the same as that in the $\left.\{110\}_{t}<\overline{1} 10\right\rangle_{t}$ twinning shown in Fig. $7(\mathrm{c})$. Therefore, it is obvious that the $\{110\}_{\mathrm{t}}\langle 1 \overline{1} 0\rangle_{\mathrm{t}}$ twinning is more difficult to occur when compared to the $\left.\{110\}_{\mathrm{t}}<\overline{1} 10\right\rangle_{\mathrm{t}}$ twinning because the $\{110\}_{\mathrm{t}}\langle 1 \overline{1} 0\rangle_{\mathrm{t}}$ twinning requires mechanism IIc together with IIb. The $\{350\}_{\mathrm{t}}<5 \overline{3} 0>_{\mathrm{t}}$ twinning involves shuffle mechanism Ic or IIa which have a very large magnitude $\Delta>0.7 a_{0}$ as shown in Fig. 7(b). Thus the $\{350\}_{\mathrm{t}}<5 \overline{3} 0>_{\mathrm{t}}$ twinning is considered not to be able to occur in practice due to the larger magnitude of shuffle. Referring to the shuffle mechanism involved in the $\{130\}_{t}<\overline{3} 10>_{t}$ twinning, mechanism Ia has 
a small magnitude in the whole range of $\delta$ as shown in Fig. 7(d). It is also apparent that the $\{130\}_{\mathrm{t}}<\overline{3} 10>_{\mathrm{t}}$ twinning is expected to occur more easily when compared to the $\{110\}_{\mathrm{t}}\langle\overline{1} 10\rangle_{\mathrm{t}}$ twinning due to the smaller magnitude of shuffle. As a result, it is concluded that the $\{130\}_{\mathrm{t}}<\overline{3} 10>_{\mathrm{t}}$ twinning is the most possible twinning mode in the tetragonal structure. The $\{130\}_{\mathrm{t}}<\overline{3} 10>_{\mathrm{t}}$ twin formed in the tetragonal structure will be observed as the $\{332\}_{\mathrm{c}}\langle 11 \overline{3}\rangle_{\mathrm{c}}$ twin in $\beta$ phase.

\subsection{Effect of the lattice modulation on the $\{332\}$ twinning}

The effect of the lattice modulation, which correspond to the shuffling of parallel adjacent $\{0 \overline{1} 1\}_{\beta}$ planes to $\langle 011\rangle_{\beta}$ directions, on twinning in the metastable $\beta$ phase is summarized in this section. In the stable bcc structure, it is reasonable that the $\{112\}_{\mathrm{c}}\langle\overline{1} \overline{1} 1\rangle_{\mathrm{c}}$ twinning is the most possible twinning mode because no shuffling is necessary for this twinning mode. However, if the lattice modulation exists in the bcc structure, the $\{112\}_{c}\langle\overline{1} \overline{1} 1\rangle_{\mathrm{c}}$ twinning is considered to become difficult to occur, since the $\{110\}_{t}\langle 1 \overline{1} 0\rangle_{t}$ twinning, which corresponds to the $\left.\{112\}_{\mathrm{c}}<\overline{1} 11\right\rangle_{\mathrm{c}}$ twinning, requires shuffles of atoms. In the modulated bcc structure, the $\{130\}_{t}<\overline{3} 10>_{t}$ twinning, which corresponds to the $\{332\}_{c}\langle 11 \overline{3}\rangle_{\mathrm{c}}$ twinning, requires a smaller magnitude of shuffle compared to the other twinning modes, and is considered as the most possible twinning mode as shown in the previous section. The most possible shuffle mechanism (Ia) of the $\{130\}_{\mathrm{t}}<\overline{3} 10>_{\mathrm{t}}$ twinning is illustrated in Fig. 8 . In this figure, $\delta$ was set equal to $1 / 24$ for example. It is clear from Fig. 8 that the $\{130\}_{\mathrm{t}}\langle\overline{3} 10\rangle_{\mathrm{t}}$ twinning requires the small and simple shuffle of atoms. We can understand the lattice correspondence between the $\{130\}_{\mathrm{t}}<\overline{3} 10>_{\mathrm{t}}$ twinning and $\{332\}_{\mathrm{c}}<11 \overline{3}>_{\mathrm{c}}$ twinning by comparing Fig. 8 and Fig. 4(b). The correspondences between the twinning planes $\left(\{130\}_{\mathrm{t}}\right.$ and $\left.\{332\}_{\mathrm{c}}\right)$ and twinning directions 
$(<\overline{3} 10\rangle_{\mathrm{t}}$ and $\left.\langle 11 \overline{3}\rangle_{\mathrm{c}}\right)$ can be seen in the twinned lattices represented by the black lines in Fig. 8 and Fig. 4(b). When we compare the magnitude of shuffle required in the $\{130\}_{t}<\overline{3} 10>_{t}$ twinning and $\{332\}_{c}<11 \overline{3}>_{c}$ twinning, the magnitude of shuffle of the $\{130\}_{t}<\overline{3} 10>_{t}$ twinning, $\Delta_{\mathrm{Ia}}=(1 / \sqrt{22}-6 \delta / \sqrt{22}) a_{0}\left(=(0.2132-1.2792 \delta) a_{0}\right)$ with $0<\delta<1 / 6$, is smaller than one-half of that of the $\{332\}_{\mathrm{c}}<11 \overline{3}>_{\mathrm{c}}$ twinning $\left(\Delta=0.4330 a_{0}\right)$. It should be noted here that if $\delta$ is set equal to 0 (bcc structure), we get $\Delta_{\mathrm{Ia}}=0.2132 a_{0}$ which does not correspond to the magnitude of shuffle of the $\{332\}_{\mathrm{c}}<11 \overline{3}>_{\mathrm{c}}$ twinning $\left(\Delta=0.4330 a_{0}\right)$. This is because that there are motif units of two atoms which are considered to shear as rigid body in the $\{130\}_{\mathrm{t}}<\overline{3} 10>_{\mathrm{t}}$ twinning (Fig. 8). If $\delta=0$, the atoms should shear as shown in Fig. 4(b) in contrast to Fig. 8 since the interplanar spacing of the planes parallel to the $K_{1}$ plane is constant in contrast to the $\delta>0$ case (Fig. $6(\mathrm{e})$ ). Therefore, when $\delta=0$, the $\{130\}_{\mathrm{t}}<\overline{3} 10>_{\mathrm{t}}$ twinning cannot be caused by the shuffle mechanism Ia with $\Delta_{\text {Ia }}=0.2132 a_{0}$ because of the different shear mechanism. When the lattice modulation exists $(\delta>0)$ due to the low shear modulus $c^{\prime}$, the decrease of $c^{\prime}$ increases $\delta$ and makes the $\{130\}_{t}<\overline{3} 10>_{t}$ twinning easier to occur. As a result, we propose that the lattice modulation in the metastable $\beta$ phase, i.e. the shuffling of parallel adjacent $\{011\}_{\beta}$ planes to $\langle 011\rangle_{\beta}$ directions, facilitates the formation of the $\{332\}_{\beta}<11 \overline{3}>_{\beta}$ twin.

\section{Conclusions}

The possible twinning modes in metastable $\beta$-type Ti alloys were discussed by considering lattice instability on the basis of the theory of the crystallography of deformation twinning proposed by Bilby and Crocker. The possibilities of the $\{332\}_{\beta}\langle 11 \overline{3}\rangle_{\beta}$ twinning and other twinning modes were compared by taking account of the shear and shuffle mechanisms of 
the twinning modes. The lattice modulation due to the low shear modulus $c^{\prime}\left(\left(c_{11}-c_{12}\right) / 2\right)$ of the $\beta$ phase, i.e. shuffling of parallel adjacent $\{0 \overline{1} 1\}_{\beta}$ planes to $\langle 011\rangle_{\beta}$ directions, was considered as the lattice instability of metastable $\beta$ phase. A tetragonal structure which consists of motif units of two atoms at each lattice point of a single base-centered tetragonal lattice was proposed for the description of the modulated bcc structure. It was found that the lattice instability makes the $\{112\}_{\beta}<\overline{1} \overline{1} 1>_{\beta}$ twinning more difficult to occur because the lattice modulation causes additional shuffling to form the $\{112\}_{\beta}<\overline{1} 11>_{\beta}$ twin. The $\{130\}_{\mathrm{t}}<\overline{3} 10>_{\mathrm{t}}$ twinning, which corresponds to the $\{332\}_{c}<11 \overline{3}>_{c}$ twinning in the bcc structure, was found to be the most possible twinning mode in the tetragonal structure because it requires a smaller magnitude of shuffle compared to the other twinning modes. In conclusion, it was proposed that the lattice modulation plays a key role to facilitate the formation of the $\{332\}_{\beta}<11 \overline{3}>_{\beta}$ twin and to suppress the formation of $\{112\}_{\beta}<\overline{1} \overline{1} 1>_{\beta}$ twin.

\section{Acknowledgements}

This work was partially supported by JSPS KAKENHI Grant Numbers 23360300 and 25289247 and by MEXT KAKENHI Grant Numbers 23102503 and 25102704.

\section{References}

[1] Christian JW, Mahajan S. Prog Mater Sci 1995;39:1.

[2] Zhu YT, Liao XZ, Wu XL. Prog Mater Sci 2012;57:1. 
[3] Vercammen S, Blanpain B, De Cooman BC, Wollants P. Acta Mater 2004;52:2005.

[4] Banerjee D, Williams JC. Acta Mater 2013;61:844.

[5] Hao YL, Li SJ, Sun SY, Zheng CY, Yang R. Acta Biomater. 2007;3:277.

[6] Tobe H, Kim HY, Miyazaki S. Mater Trans 2009;50:2721.

[7] Niinomi M. J Mech Behav Biomed Mater 2008;1:30.

[8] Kim HY, Ikehara Y, Kim JI, Hosoda H, Miyazaki S. Acta Mater 2006;54:2419.

[9] Kawabe Y, Muneki S. ISIJ Int 1991;31:785.

[10] Furuhara T. Met Mater 2000;6:221.

[11] Ivasishin OM, Markovsky PE, Matviychuk YV, Semiatin SL, Ward CH, Fox S. J Alloys Compd 2008;457:296.

[12] Min XH, Tsuzaki K, Emura S, Tsuchiya K. Mater Sci Eng A 2011;528:4569.

[13] Min XH, Tsuzaki K, Emura S, Tsuchiya K. Mater Sci Eng A 2012;554:53.

[14] Min X, Chen X, Emura S, Tsuchiya K. Scripta Mater 2013;69:393.

[15] Hanada S, Yoshio T, Izumi O. Trans Jpn Inst Met 1986;27:496.

[16] Hanada S, Izumi O. Metall Trans A 1987;18A:265.

[17] Sun F, Zhang JY, Marteleur M, Gloriant T, Vermaut P, Laillé D, et al. Acta Mater $2013 ; 61: 6406$.

[18] Hanada S, Izumi O. Metall Trans A 1986;17A:1409.

[19] Furuhara T, Kishimoto K, Maki T. Mater Trans 1994;35:843.

[20] Zhao X, Niinomi M, Nakai M, Miyamoto G, Furuhara T. Acta Biomater 2011;7:3230.

[21] Saito T, Furuta T, Hwang JH, Kuramoto S, Nishino K, Suzuki N, et al. Science $2003 ; 300: 464$.

[22] Kuramoto S, Furuta T, Hwang JH, Nishino K, Saito T. Metall Mater Trans A 2006;37A:657. 
[23] Gutkin MY, Ishizaki T, Kuramoto S, Ovid'ko IA. Acta Mater 2006;54:2489.

[24] Gutkin MY, Ishizaki T, Kuramoto S, Ovid'ko IA, Skiba NV. Int J Plast 2008;24:1333.

[25] Withey EA, Minor AM, Chrzan DC, Morris Jr JW, Kuramoto S. Acta Mater 2010;58:2652.

[26] Kuramoto S, Furuta T, Hwang J, Nishino K, Saito T. J Jpn Inst Met 2005;69:953.

[27] Yang Y, Li GP, Cheng GM, Li YL, Yang K. Appl Phys Lett 2009;94:061901.

[28] Yang Y, Wu SQ, Li GP, Li YL, Lu YF, Yang K, et al. Acta Mater 2010;58:2778.

[29] Qu L, Yang Y, Lu YF, Feng L, Ju JH, Ge P, et al. Scripta Mater 2013;69:389.

[30] Blackburn MJ, Feeney JA. J Inst Met 1971;99:132.

[31] Carter G, Flower HM, Pennock GM, West DRF. J Mater Sci 1977;12:2149.

[32] Hanada S, Izumi O. Metall Trans A 1980;11A:1447.

[33] Hida M, Sukedai E, Henmi C, Sakaue K, Terauchi H. Acta Metall 1982;30:1471.

[34] Min XH, Emura S, Nishimura T, Tsuchiya K, Tsuzaki K. Mater Sci Eng A 2010;527:5499.

[35] Oka M, Taniguchi Y. Metall Trans A 1979;10A:651.

[36] Hanada S, Takemura A, Izumi O. Trans Jpn Inst Met 1982;23:507.

[37] Hanada S, Ozeki M, Izumi O. Metall Trans A 1985;16A:789.

[38] Kawabata T, Kawasaki S, Izumi O. Acta Mater 1998;46:2705.

[39] Bertrand E, Castany P, Péron I, Gloriant T. Scripta Mater 2011;64:1110.

[40] Hanada S, Izumi O. J Mater Sci 1986;21:4131.

[41] Zhao X, Niinomi M, Nakai M, Hieda J, Ishimoto T, Nakano T. Acta Biomater 2012;8:2392.

[42] Hanada S, Yoshio T, Izumi O. J Mater Sci 1986;21:866.

[43] Crocker AG. Acta Metall 1962;10:113.

[44] Richman RH. In: Reed-Hill RE, Hirth JP, Rogers HC, editors. Deformation Twinning. New York: Gordon and Breach; 1964. p. 237.

[45] Takemoto Y, Hida M, Sakakibara A. J Jpn Inst Met 1993;57:1471. 
[46] Litvinov VS, Rusakov GM. Phys Met Metallogr 2000;90:96.

[47] Mantani Y, Takemoto Y, Hida M, Sakakibara A. J Jpn Inst Met 2002;66:1022.

[48] Oberson PG, Ankem S. Phys Rev Lett 2005;95:165501.

[49] Oka M, Taniguchi Y. J Jpn Inst Met 1978;42:814.

[50] Bilby BA, Crocker AG. Proc R Soc A 1965;288:240.

[51] Crocker AG. J Nucl Mater 1965;16:306.

[52] Nii Y, Arima T, Kim HY, Miyazaki S. Phys Rev B 2010;82:214104.

[53] Tahara M, Kim HY, Inamura T, Hosoda H, Miyazaki S. Acta Mater 2011;59:6208.

[54] Kim HY, Wei L, Kobayashi S, Tahara M, Miyazaki S. Acta Mater 2013;61:4874.

[55] Moffat DL, Larbalestier DC. Metall Trans A 1988;19A:1677.

[56] Banumathy S, Mandal RK, Singh AK. J Appl Phys 2009;106:093518.

[57] Obbard EG, Hao YL, Talling RJ, Li SJ, Zhang YW, Dye D, et al. Acta Mater 2011;59:112.

[58] Li CX, Luo HB, Hu QM, Yang R, Yin FX, Umezawa O, et al. Solid State Commun 2013;159:70. 


\section{List of figures}

Fig. 1. Twinning modes with $q=1$ (a), $q=2$ (b), $q=3$ (c) and $q=4$ (d) in single lattice structures. The red dotted arrows indicate the shear of the lattice points, and the green dotted circles indicate the lattice points of the twin. No shuffling is necessary to produce the twin when $q=1$ or 2.

Fig. 2. Twinning mode with $q=1$ in a double lattice structure. Shear of the lattice points (a) and shuffle mechanisms correspond to the rearrangement of the motif units required to produce the type I twin (b) and type II twin (c). The atoms represented by open and closed circles lie above and below the paper, respectively.

Fig. 3 Twinning mode with $q=4$ in a double lattice structure. Shear of the lattice points (a) and shuffle mechanisms correspond to the disruption of the motif units required to produce the type I twin (b and c) and type II twin (d and e). The rearrangement of the motif units is also required at one-half of the lattice points by means of one of the mechanisms shown in Fig. 2. The atoms represented by open and closed circles lie above and below the paper, respectively.

Fig. 4. (11 0$)$ projection of the (112) [11 $]$ twinning (a) and (332) $[11 \overline{3}]$ twinning (b) in the bcc structure. Closed circles represent atoms in the projection plane, and open circles are atoms $0.7071 a_{0}$ above or below the projection. The red and green arrows indicate the shear and shuffle, respectively.

Fig. 5. Schematic diagram of the bcc structure (a) and the modulated bcc structure (b). The modulated structure can be treated as the tetragonal structure shown in (c) or (d). The orange 
arrows indicate the shift of the atoms due to the modulation. The blue lines indicate motif units of two atoms.

Fig. 6. (001) $)_{\mathrm{t}}$ projection of the motif units (a), and the possible motif unit of the $(110)_{\mathrm{t}}\left[\begin{array}{ll}1 & 1\end{array}\right]_{\mathrm{t}}$ twinning (b), $\quad(350)_{\mathrm{t}}[5 \overline{3} 0]_{\mathrm{t}}$ twinning (c), $\quad(110)_{\mathrm{t}}[\overline{1} 10]_{\mathrm{t}}$ twinning (d) and $(130)_{\mathrm{t}}[\overline{3} 10]_{\mathrm{t}}$ twinning (e). Closed circles represent atoms in the projection plane, and open circles are atoms $0.7071 a_{0}$ above or below the projection. The red lines indicate the possible twinning planes and directions.

Fig. 7. Magnitude of shuffle normalized by the lattice constant $\left(\Delta / a_{0}\right)$ of the $(110)_{\mathrm{t}}\left[\begin{array}{ll}1 & \overline{1}\end{array}\right]_{\mathrm{t}}$ twinning $(q=4)(\mathrm{a}),(350)_{\mathrm{t}}[5 \overline{3} 0]_{\mathrm{t}}$ twinning $(q=4)(\mathrm{b}),(110)_{\mathrm{t}}[\overline{1} 10]_{\mathrm{t}} \quad$ twinning $(q=2)(\mathrm{c})$ and $(130)_{\mathrm{t}}[\overline{3} 10]_{\mathrm{t}}$ twinning $(q=2)(\mathrm{d})$ as a function of $\delta$.

Fig. 8. $(001)_{\mathrm{t}}$ projection of the $(130)_{\mathrm{t}}[\overline{3} 10]_{\mathrm{t}}$ twinning in the tetragonal structure $(\delta=1 / 24)$. Closed circles represent atoms in the projection plane, and open circles are atoms $0.7071 a_{0}$ above or below the projection. The red arrows indicate the shear of the lattice points. The blue lines and arrows indicate the motif units and the shear of the atoms comprising the units, respectively. The green arrows indicate the shuffle. 
List of tables

Table 1

Possible twinning modes in the bcc structure.

Table 2

Six lattice correspondence variants derived from the tetragonal and cubic structures.

Table 3

Possible twinning modes in the tetragonal structure.

Table 4

Possible motif units: $[x y z]_{\mathrm{t}}$.

Table 5

Suggested shuffle mechanisms. 


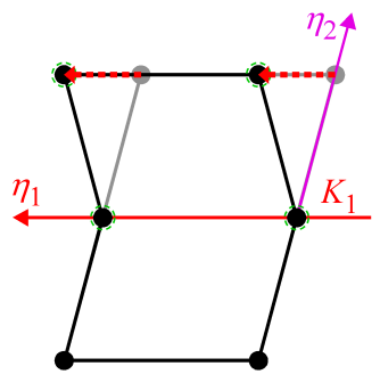

(a)

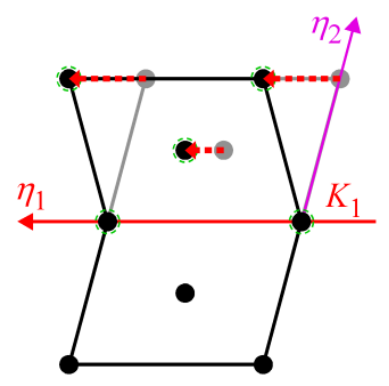

(b)

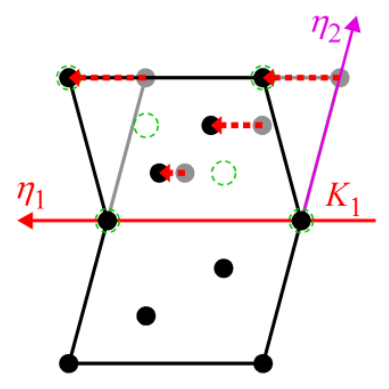

(c)

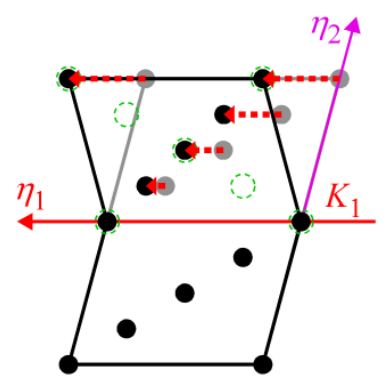

(d)

Fig. 1 


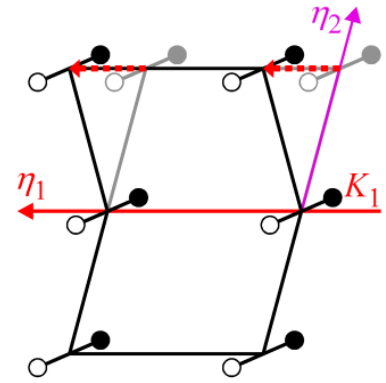

(a)

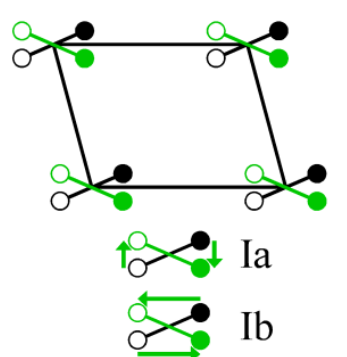

(b)

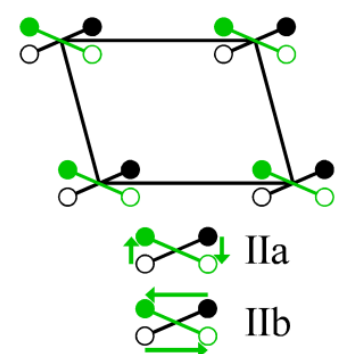

(c)

Fig. 2 


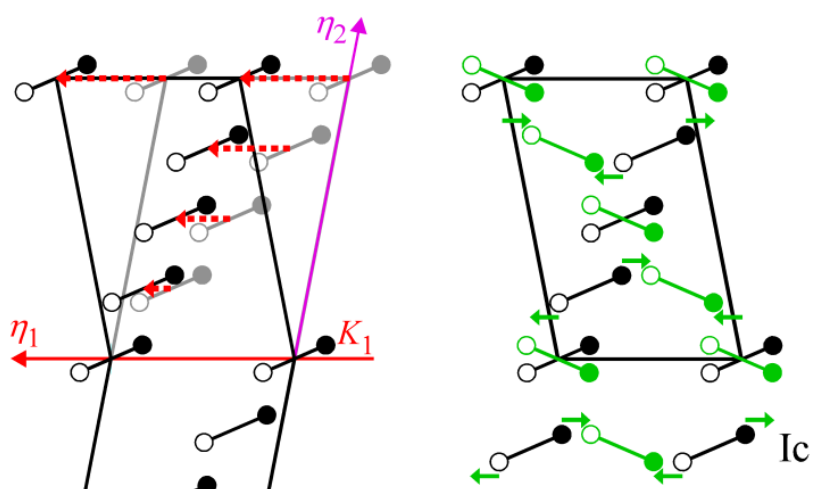

(b)

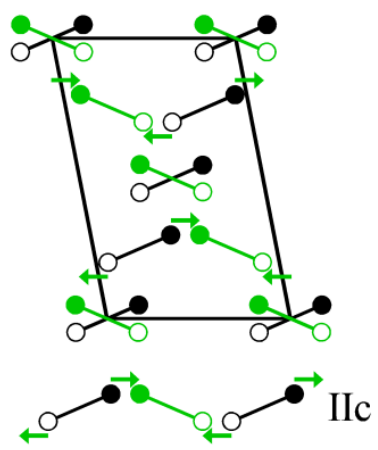

(d)

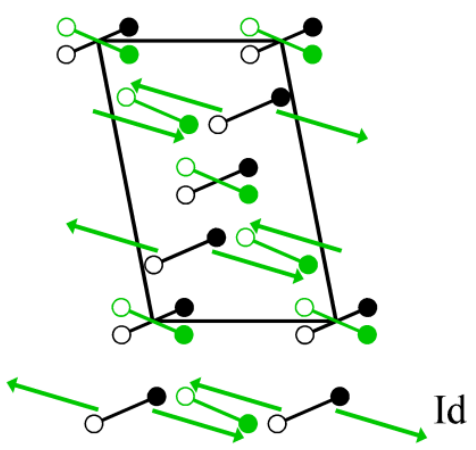

(c)
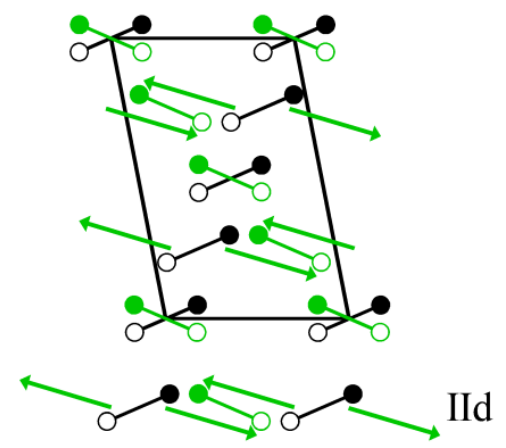

(e)

Fig. 3 


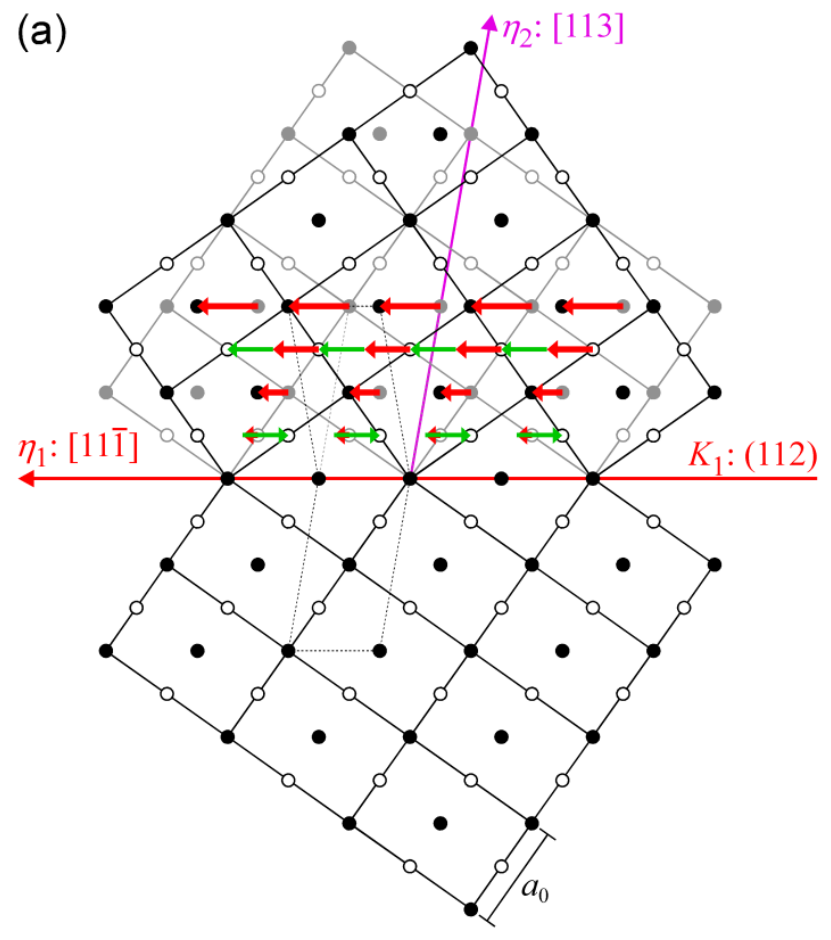

(b)

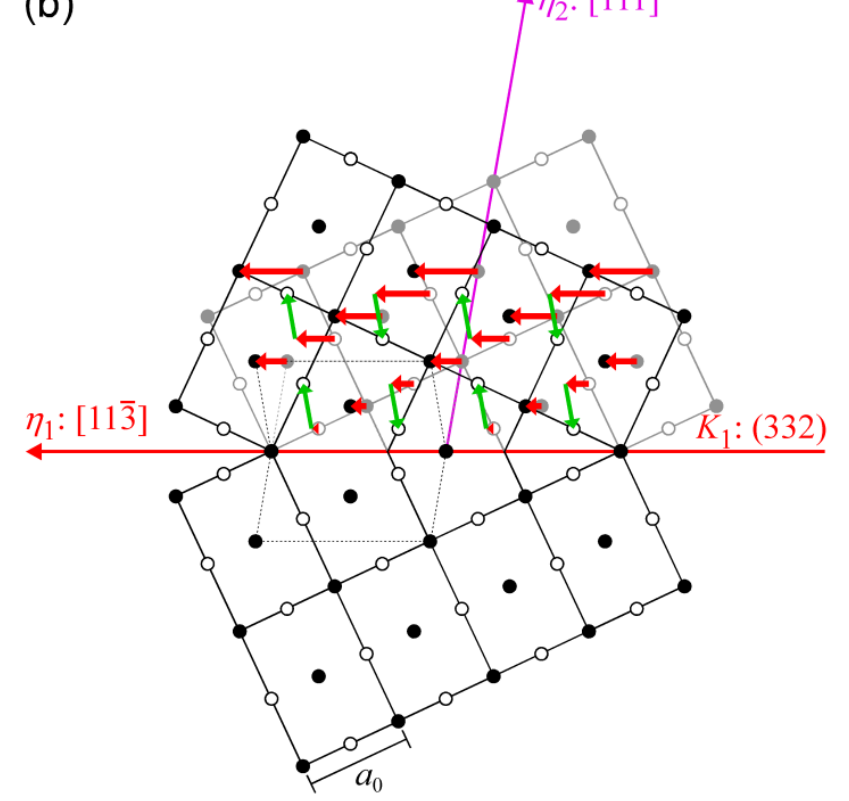

Fig. 4 


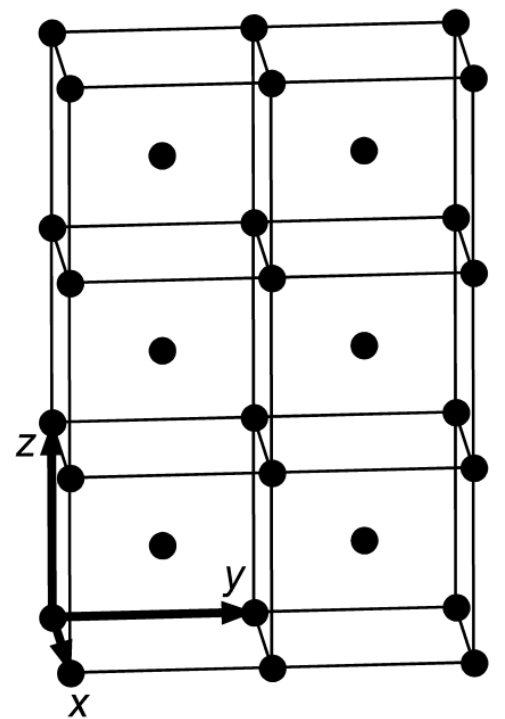

(a)

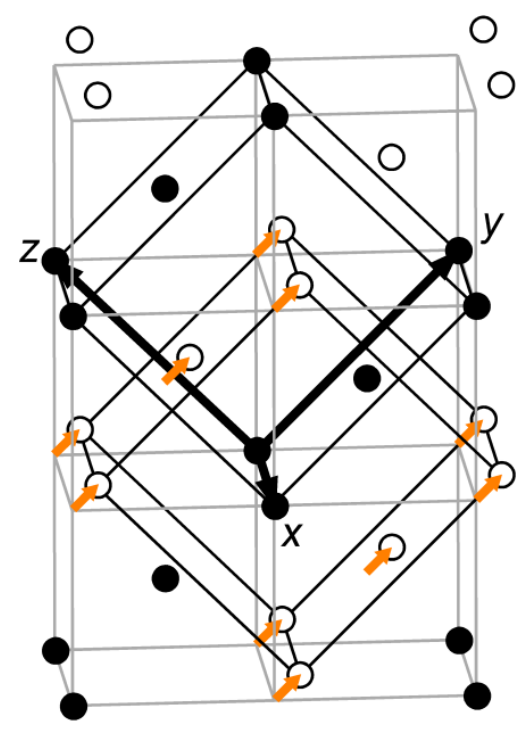

(c)

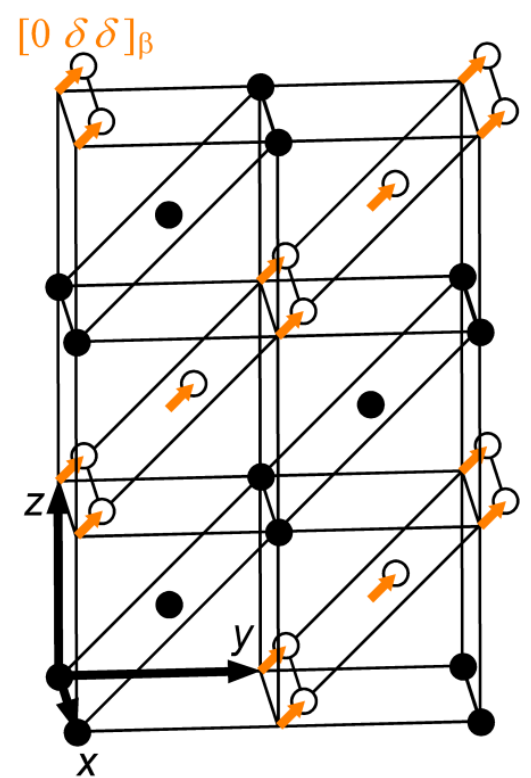

(b)

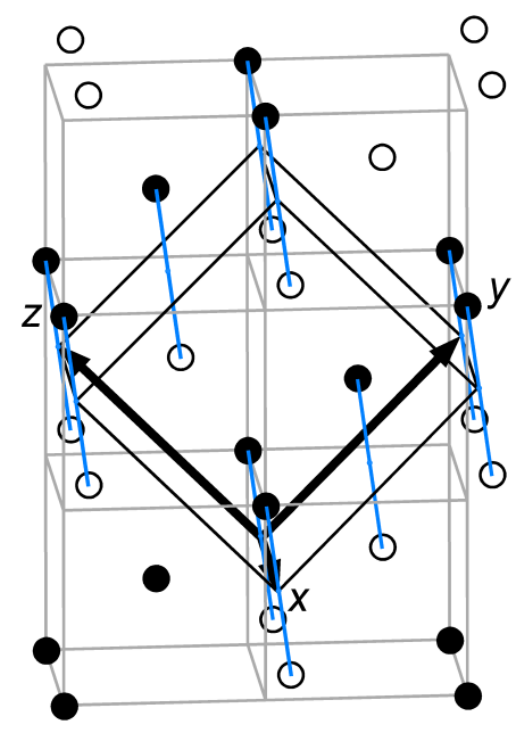

(d)

Fig. 5 


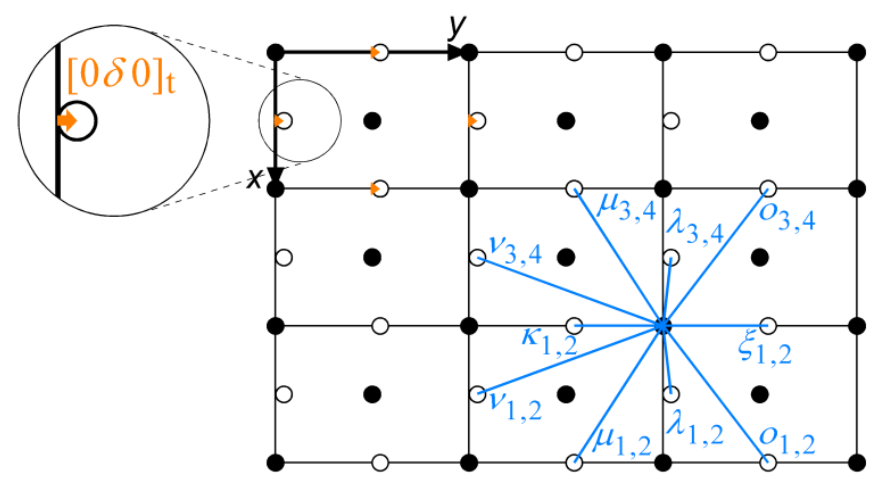

(a)

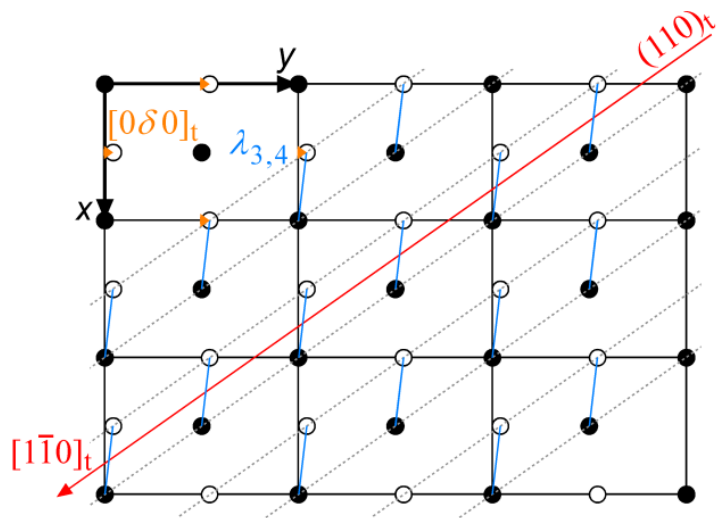

(b)

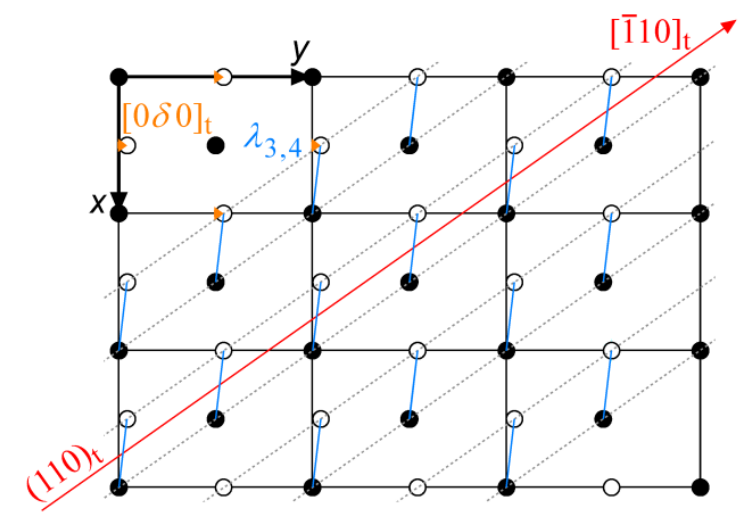

(d)

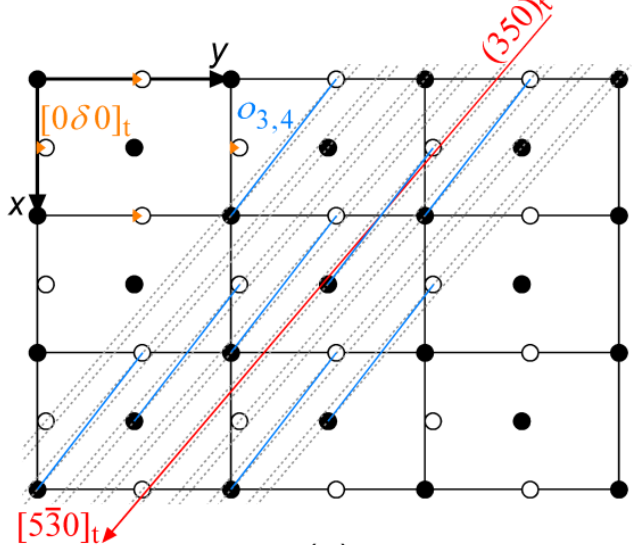

(c)

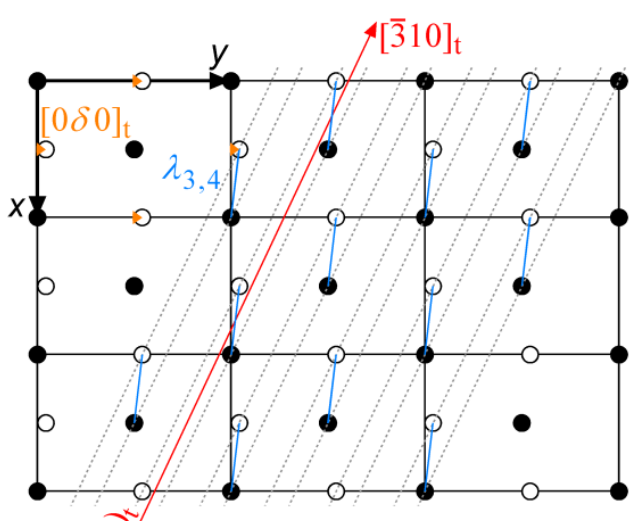

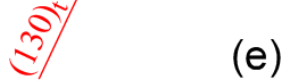

Fig. 6 


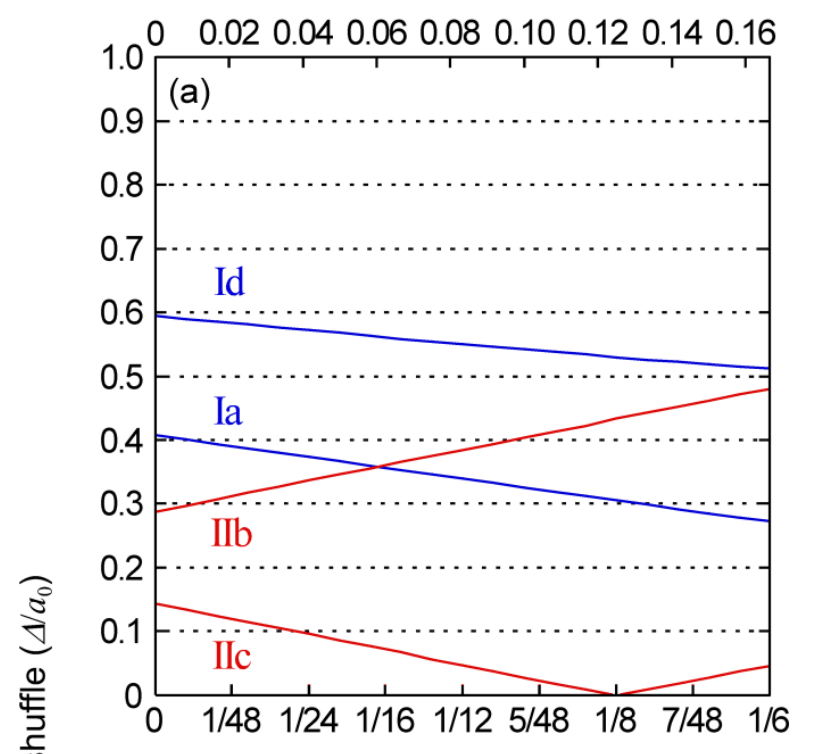

$\begin{array}{lllllllllllllll}0 & 0.02 & 0.04 & 0.06 & 0.08 & 0.10 & 0.12 & 0.14 & 0.16\end{array}$
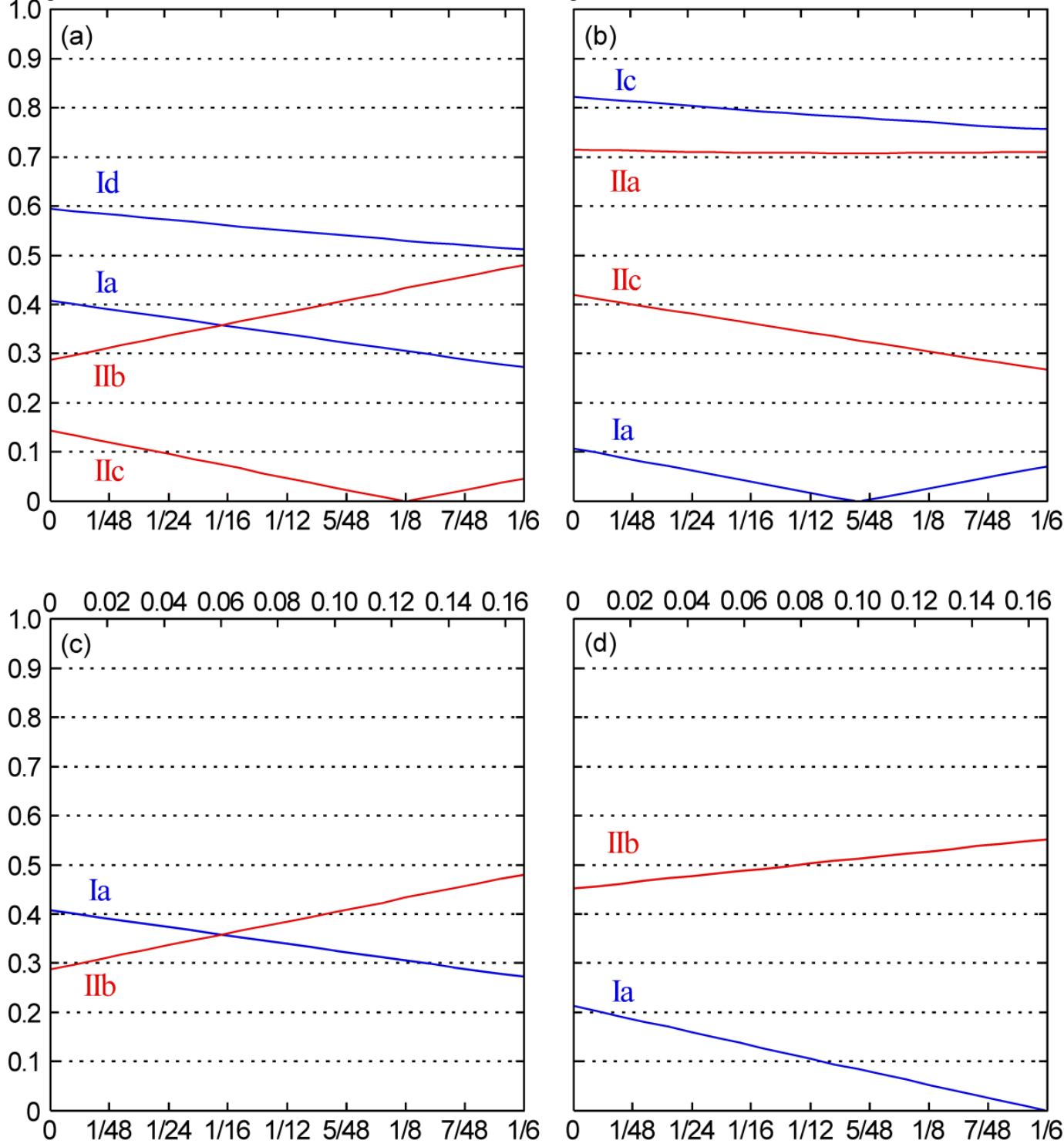

$\begin{array}{llllllllllllllllllll}0 & 0.02 & 0.04 & 0.06 & 0.08 & 0.10 & 0.12 & 0.14 & 0.16\end{array}$

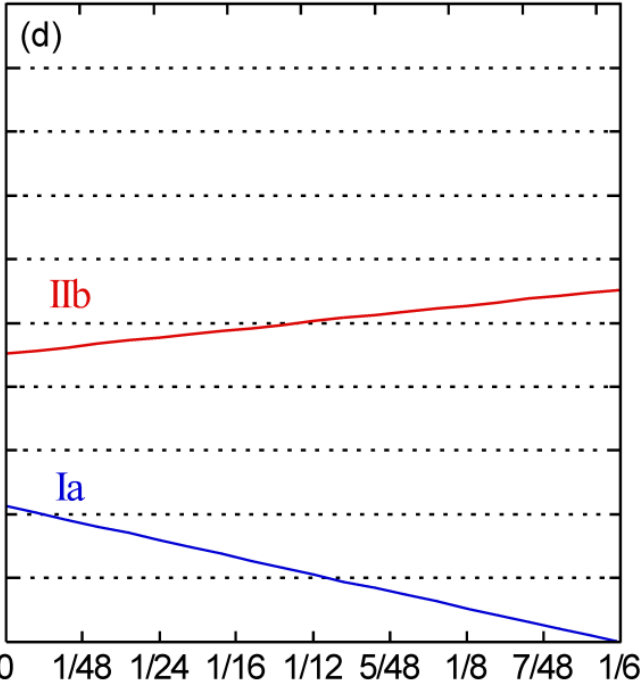

$\delta$

Fig. 7 


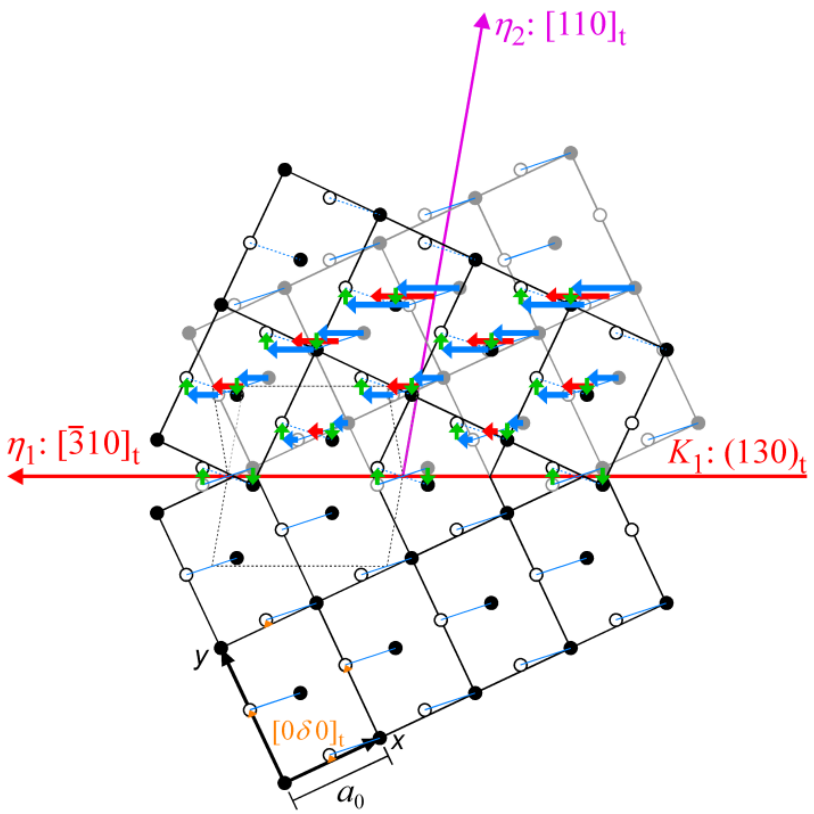

Fig. 8 
Table 1

\begin{tabular}{lllllll}
\hline Mode $s$ & $K_{1}$ & $K_{2}$ & $\eta_{1}$ & $\eta_{2}$ & $q$ \\
\hline
\end{tabular}

$1 \quad 0.3536 \quad 112 \quad 33 \overline{2} \quad\langle 11 \overline{1}\rangle \quad\langle 113\rangle \quad 4$

$2 \quad \begin{array}{llllll}0.3536 & 332 & 1 \overline{2} & \langle 1 \overline{3}\rangle & \langle 111\rangle & 4\end{array}$

$3 \quad 0.6124 \quad 110 \quad \overline{5}, 11,8 \quad\langle\overline{1} 11\rangle \quad\langle 53 \overline{1}\rangle \quad 4$

$4 \quad \begin{array}{llllll}0.6124 & 5,11,8 & \overline{1} 10 & \langle\overline{5} 3 \overline{1}\rangle & \langle 111\rangle & 4\end{array}$

$\begin{array}{lllllll}5 & 0.7071 & 112 & \overline{1} 12 & \langle\overline{1} 11\rangle & \langle 111\rangle & 2\end{array}$

$6 \quad 0.7071 \quad 110 \quad 114 \quad\langle 001\rangle\langle 22 \overline{1}\rangle 4$

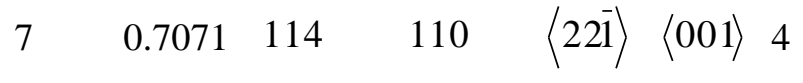


Table 2

\begin{tabular}{lll}
\hline Variant $[100]_{\mathrm{t}}$ & {$[010]_{\mathrm{t}}$} & {$[001]_{\mathrm{t}}$} \\
\hline
\end{tabular}

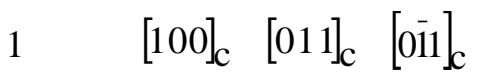

$2 \quad[100]_{\mathcal{C}}[011]_{\mathfrak{C}} \quad[011]_{\mathfrak{C}}$

$3 \quad[010]_{\mathrm{c}}[101]_{\mathrm{C}} \quad[101]_{\mathrm{c}}$

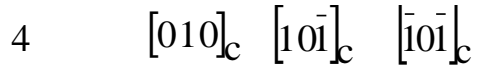

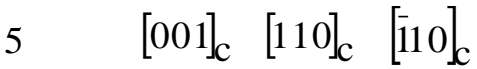

$6 \quad[001]_{\mathcal{C}}[\overline{110}]_{\mathcal{C}}[\overline{11} 0]_{\mathcal{C}}$ 


\section{Base-centered tetragonal} Corresponding body-centered cubic

\section{mans.} $K_{1} \quad K_{2}$

$10.1768 \quad 110_{\mathrm{t}}$

$K_{2} \quad \eta_{1}$

$\eta_{2}$
$q K_{1}$ $K_{2}$ $\eta_{1}$ $\eta_{2}$ $\overline{5} \overline{5} 6_{\mathrm{c}}$

$\langle\overline{i i 1}\rangle_{\mathrm{c}}$ $\langle 335\rangle_{\mathrm{c}} \quad 8$

20.1768

$350_{\mathrm{t}}$ $110_{\mathrm{t}}$ $\langle 5 \overline{3} 0\rangle_{\mathrm{t}} \quad\langle 110\rangle_{\mathrm{t}} \quad 4 \quad 556_{\mathrm{c}} \quad \overline{11} 2_{\mathrm{c}}$ $\langle\overline{3} 35\rangle_{\mathrm{c}}$ $\langle 111\rangle_{\mathrm{c}} \quad 8$

$30.3062(001)_{\mathrm{t}}$

$$
8, \overline{16}, 3 \mathrm{t}
$$

$\langle 110\rangle_{\mathrm{t}}$

$\langle\overline{118}\rangle_{\mathrm{t}} \quad 4 \quad 110_{\mathrm{c}}$

$\overline{13}, 19,16_{\mathrm{c}}\langle\overline{11} 1\rangle_{\mathrm{c}}$

$\langle 971\rangle_{\mathrm{c}} \quad 8$

$4 \quad 0.30628,16,3 \mathrm{t} \quad(001)_{\mathrm{t}}$

$\langle\overline{11} 18\rangle_{\mathrm{t}} \quad\langle 110\rangle_{\mathrm{t}} \quad 4 \quad 13,19,16_{\mathrm{c}} \quad \overline{11}_{\mathrm{c}}$

$\langle\overline{971}\rangle_{\mathrm{c}} \quad\langle 111\rangle_{\mathrm{c}} \quad 8$

$\begin{array}{lll}5 & 0.3536 & 110_{\mathrm{t}}\end{array}$ $\overline{130}_{\mathrm{t}}$ $\langle\overline{110}\rangle_{\mathrm{t}} \quad\langle 310\rangle_{\mathrm{t}} \quad 2 \quad 112 \mathrm{c} \quad 33 \overline{\mathrm{c}}$

$\langle 11 \overline{1}\rangle_{\mathrm{c}}$ $\langle 113\rangle_{\mathrm{c}} \quad 4$

$\begin{array}{lll}6 & 0.3536 & 130_{\mathrm{t}}\end{array}$ $\overline{11}_{\mathrm{t}}$ $\langle\overline{3} 10\rangle_{\mathrm{t}} \quad\langle 110\rangle_{\mathrm{t}} \quad 2 \quad 332 \mathrm{c} \quad 11 \overline{2}_{\mathrm{c}}$ $\langle 11 \overline{3}\rangle_{\mathrm{c}} \quad\langle 111\rangle_{\mathrm{c}} \quad 4$

$7 \quad 0.3536 \quad 113_{t}$

$$
3 \overline{5} 1_{\mathrm{t}}
$$

$\langle 110\rangle_{\mathrm{t}} \quad\langle 112\rangle_{\mathrm{t}} \quad 4 \quad 112 \mathrm{c} \quad 33 \overline{\mathrm{c}}$

$\langle 11 \overline{1}\rangle_{\mathrm{c}}$ $\langle 113\rangle_{\mathrm{c}} \quad 4$

$8 \quad 0.3536 \quad 351_{\mathrm{t}} \quad 113 \mathrm{t}$ $\langle 112\rangle_{\mathrm{t}} \quad\langle 110\rangle_{\mathrm{t}} \quad 4 \quad 332 \mathrm{c} \quad 11 \overline{2}_{\mathrm{c}}$ $\langle 11 \overline{3}\rangle_{\mathrm{c}} \quad\langle 111\rangle_{\mathrm{c}} \quad 4$ $9 \quad 0.3536(001)_{\mathrm{t}}$ $40 \overline{1}_{\mathrm{t}} \quad[100]_{\mathrm{t}}$ $\langle\overline{10} \overline{4}\rangle_{\mathrm{t}} \quad 4 \quad 110_{\mathrm{c}}$ $118_{\mathrm{c}}$ $\langle 001\rangle_{\mathrm{c}} \quad\langle 44 \overline{1}\rangle_{\mathrm{c}} \quad 8$ $10 \quad 0.3536 \quad 401_{t}$ $11 \quad 0.3953 \quad 112_{\mathrm{t}}$ $(00)_{t}^{-}$ $\langle\overline{104}\rangle_{\mathrm{t}} \quad[100]_{\mathrm{t}} \quad 4 \quad 118_{\mathrm{c}}$ $110 \mathrm{c}$ $\langle 44 \overline{1}\rangle_{\mathrm{c}} \quad\langle 001\rangle_{\mathrm{c}} \quad 8$ $120.3953 \quad 536_{\mathrm{t}}$ $\overline{5}^{3} 6_{\mathrm{t}}$ $\langle\overline{5} 12\rangle_{\mathrm{t}}$

$$
\langle 312\rangle_{\mathrm{t}} \quad 4 \quad 321_{\mathrm{c}}
$$

$9, \overline{10}, 3 \mathrm{c}$

$\langle 3 \overline{5} 1\rangle_{\mathrm{c}}$ $\langle 331\rangle_{\mathrm{c}} \quad 8$ $112_{\mathrm{t}}$

$\langle 512\rangle_{\mathrm{t}} \quad 4 \quad 9,10,3 \mathrm{c}$

$3 \overline{2} 1_{\mathrm{c}}$

$\langle 3 \overline{3} 1\rangle_{\mathrm{c}} \quad\langle 351\rangle_{\mathrm{c}} \quad 8$

$13 \quad 0.4678 \quad 021_{\mathrm{t}}$ $\overline{86} \overline{5}_{\mathrm{t}}$ $\langle\overline{5} 1 \overline{2}\rangle_{\mathrm{t}} \quad\langle 132\rangle_{\mathrm{t}} \quad 4 \quad 310_{\mathrm{c}}$

$1,11, \overline{16}_{\mathrm{c}}$ $\langle\overline{135}\rangle_{\mathrm{c}} \quad\langle 511\rangle_{\mathrm{c}} \quad 8$ $14 \quad 0.4678 \quad 865$ t $02 \overline{1}_{\mathrm{t}}$ $\langle\overline{13} \overline{2}\rangle_{\mathrm{t}}$ $\langle 512\rangle_{\mathrm{t}} \quad 4 \quad 1,11,16_{\mathrm{c}}$ $310_{c}$ $\langle 511\rangle_{\mathrm{c}} \quad\langle 135\rangle_{\mathrm{c}} \quad 8$ $15 \quad 0.4678 \quad 261_{t}$ $\begin{array}{lll}16 & 0.4678 & 223 \\ \mathrm{t}\end{array}$ $\overline{2}_{2} 3_{\mathrm{t}}$ $\langle\overline{13}, 3,8\rangle_{\mathrm{t}}\langle 110\rangle_{\mathrm{t}} \quad 4 \quad 754_{\mathrm{c}} \quad 51 \overline{4}_{\mathrm{c}}$ $\langle 11, \overline{5}, \overline{13}\rangle_{\mathrm{c}}\langle 111\rangle_{\mathrm{c}} \quad 8$ $17 \quad 0.5000(001)_{\mathrm{t}}$ $\overline{2}^{6} 1_{\mathrm{t}}$ $\langle\overline{110}\rangle_{\mathrm{t}} \quad\langle 13,3,8\rangle_{\mathrm{t}} \quad 4 \quad 514_{\mathrm{c}} \quad 7 \overline{5}_{\mathrm{c}}$ $\langle 111\rangle_{\mathrm{c}}$ $\langle 11,5,13\rangle_{\mathrm{c}} 8$

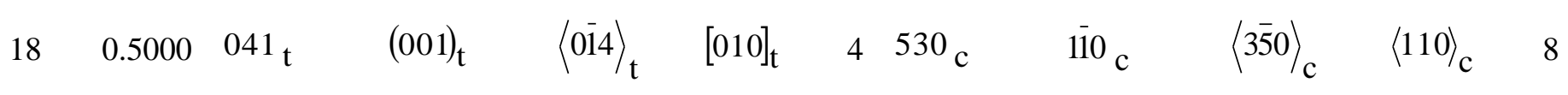




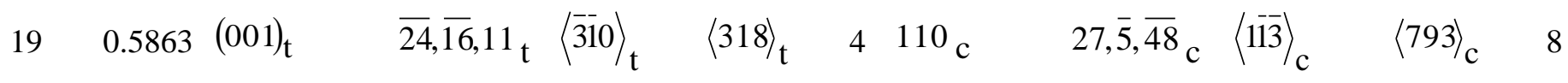

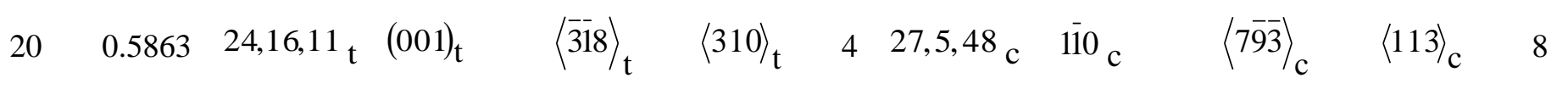

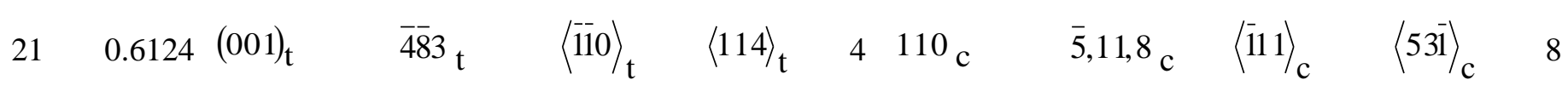
$\begin{array}{llllllllllll}22 & 0.6124 & 483_{\mathrm{t}} & (001)_{\mathrm{t}} & \langle\overline{1} 14\rangle_{\mathrm{t}} & \langle 110\rangle_{\mathrm{t}} & 4 & 5,11,8_{\mathrm{c}} & \overline{110} \mathrm{c} & \langle\overline{5} 3 \overline{1}\rangle_{\mathrm{c}} & \langle 111\rangle_{\mathrm{c}} & 8\end{array}$

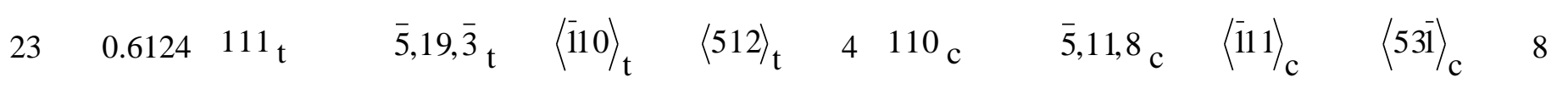
$\begin{array}{llllllllllll}24 & 0.6124 & 5,19,3_{\mathrm{t}} & \overline{1} 1 \overline{1}_{\mathrm{t}} & \langle\overline{5} 1 \overline{2}\rangle_{\mathrm{t}} & \langle 110\rangle_{\mathrm{t}} & 4 & 5,11,8_{\mathrm{c}} & \overline{11} 0_{\mathrm{c}} & \langle\overline{5} 3 \overline{1}\rangle_{\mathrm{c}} & \langle 111\rangle_{\mathrm{c}} & 8\end{array}$ $25 \quad 0.6847 \quad 023{ }_{\mathrm{t}} \quad \overline{8} 61_{\mathrm{t}} \quad\langle\overline{13}, 3, \overline{2}\rangle_{\mathrm{t}}\langle 112\rangle_{\mathrm{t}} \quad 4 \quad 510_{\mathrm{c}} \quad 7, \overline{5}, \overline{16} \mathrm{c} \quad\langle 1, \overline{5}, \overline{13}\rangle_{\mathrm{c}} \quad\langle 311\rangle_{\mathrm{c}} \quad 8$

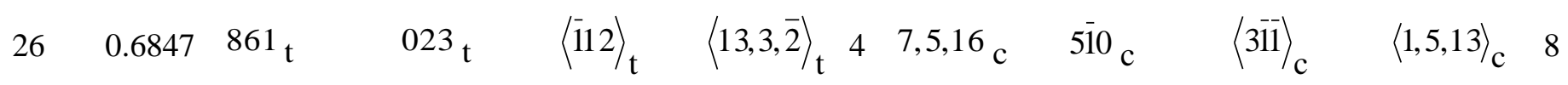
$27 \quad 0.6847 \quad 221_{\mathrm{t}} \quad \overline{2}, 6,11_{\mathrm{t}} \quad\langle\overline{738}\rangle_{\mathrm{t}} \quad\langle 310\rangle_{\mathrm{t}} \quad 4 \quad 431_{\mathrm{c}} \quad \overline{4}, 17, \overline{5}_{\mathrm{c}} \quad\langle\overline{7}, 11, \overline{5}\rangle_{\mathrm{c}} \quad\langle 311\rangle_{\mathrm{c}} \quad 8$

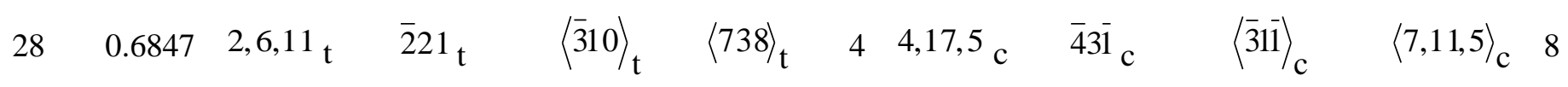

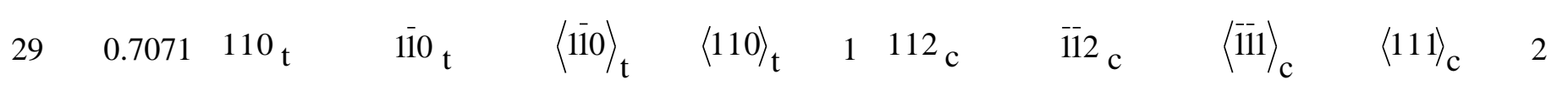

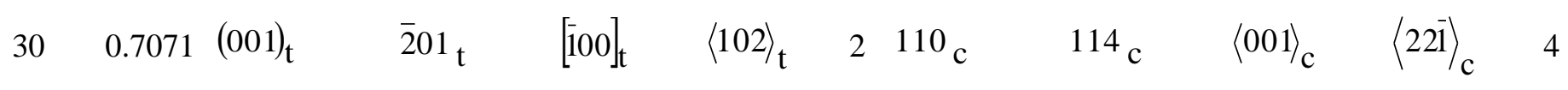

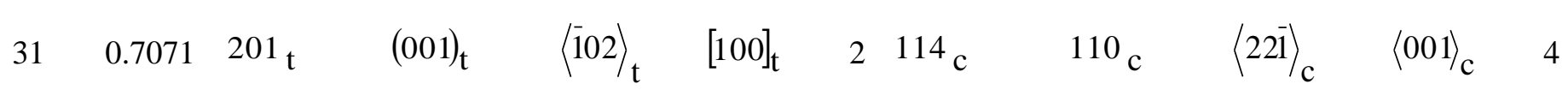

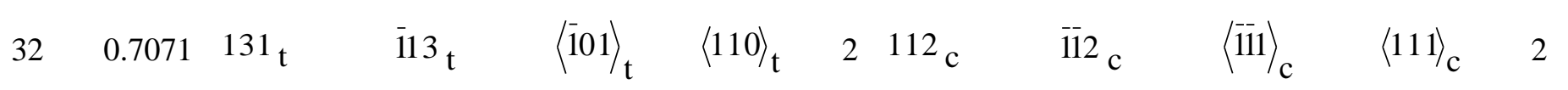
$\begin{array}{llllllllllll}33 & 0.7071 & 113_{\mathrm{t}} & \overline{13} 1_{\mathrm{t}} & \langle\overline{11} 0\rangle_{\mathrm{t}} & \langle 101\rangle_{\mathrm{t}} & 4 & 112 \mathrm{c} & \overline{11} 2_{\mathrm{c}} & \langle\overline{111}\rangle_{\mathrm{c}} & \langle 111\rangle_{\mathrm{c}} & 2\end{array}$

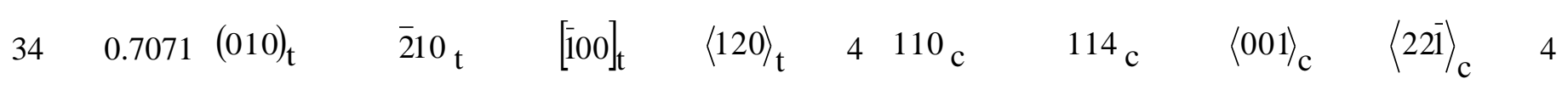
$\begin{array}{llllllllllll}35 & 0.7071 & 210_{\mathrm{t}} & (010)_{\mathrm{t}} & \langle\overline{120}\rangle_{\mathrm{t}} & {[100]_{\mathrm{t}}} & 4 & 114_{\mathrm{c}} & 110_{\mathrm{c}} & \langle 22 \overline{1}\rangle_{\mathrm{c}} & \langle 001\rangle_{\mathrm{c}} & 4\end{array}$

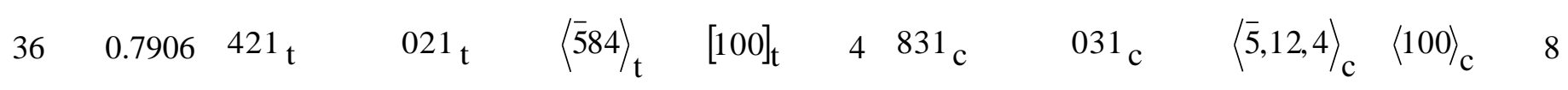
$\begin{array}{lllllllllll}37 & 0.7906 & 021_{\mathrm{t}} & 421_{\mathrm{t}} & {[100]_{\mathrm{t}}} & \langle\overline{5} 84\rangle_{\mathrm{t}} & 4 & 031_{\mathrm{c}} & 831_{\mathrm{c}} & \langle 100\rangle_{\mathrm{c}} & \langle\overline{5}, 12,4\rangle_{\mathrm{c}}\end{array}$ 
Table 4

\begin{tabular}{|c|c|c|c|}
\hline Unit & & $y$ & $z$ \\
\hline$\lambda_{1}$ & $1 / 2$ & $\delta$ & $1 / 2$ \\
\hline$\lambda_{2}$ & $1 / 2$ & $\delta$ & $1 / 2$ \\
\hline$\lambda_{3}$ & $1 / 2$ & $\delta$ & $1 / 2$ \\
\hline$\lambda_{4}$ & $1 / 2$ & $\delta$ & $1 / 2$ \\
\hline$\kappa_{1}$ & 0 & $\delta 1 / 2$ & $1 / 2$ \\
\hline$\kappa_{2}$ & 0 & $\delta 1 / 2$ & $1 / 2$ \\
\hline$\xi_{1}$ & 0 & $\delta+1 / 2$ & $1 / 2$ \\
\hline$\xi_{2}$ & 0 & $\delta+1 / 2$ & $1 / 2$ \\
\hline$\mu_{1}$ & 1 & $\delta 1 / 2$ & $1 / 2$ \\
\hline$\mu_{2}$ & 1 & $\delta 1 / 2$ & $1 / 2$ \\
\hline$\mu_{3}$ & 1 & $\delta 1 / 2$ & $1 / 2$ \\
\hline$\mu_{4}$ & & $\delta 1 / 2$ & $1 / 2$ \\
\hline$o_{1}$ & & $\delta+1 / 2$ & $1 / 2$ \\
\hline$o_{2}$ & 1 & $\delta+1 / 2$ & $1 / 2$ \\
\hline$o_{3}$ & 1 & $\delta+1 / 2$ & $1 / 2$ \\
\hline$o_{4}$ & & $\delta+1 / 2$ & $1 / 2$ \\
\hline$v_{1}$ & $1 / 2$ & 01 & 172 \\
\hline$v_{2}$ & $1 / 2$ & $\delta 1$ & $1 / 2$ \\
\hline$v_{3}$ & $1 / 2$ & $\delta 1$ & $1 / 2$ \\
\hline$v_{4}$ & $1 / 2$ & $\delta 1$ & $1 / 2$ \\
\hline
\end{tabular}


Table 5

Mode $K_{1} \quad \eta_{1} \quad q$ Unit Mechanism

Type I Type II

$1 \quad 110_{\mathrm{t}}\langle 110\rangle_{\mathrm{t}} 4 \lambda_{3,4}$ Ia $\quad$ IIb

\begin{tabular}{rrrrr} 
& & Id & IIc \\
\hline 2 & $350 \mathrm{t}\langle 5 \overline{3} 0\rangle_{\mathrm{t}} 4$ & $o_{3,4}$ & Ia & IIa \\
& & Ic & IIc \\
\hline
\end{tabular}

$\begin{array}{lllllll}5 & 110_{\mathrm{t}} & \langle 110\rangle_{\mathrm{t}} & 2 & \lambda_{3,4} & \text { Ia } & \text { IIb }\end{array}$

$6 \quad 130 \mathrm{t}\langle\overline{310}\rangle_{\mathrm{t}} 2 \lambda_{3,4}$ Ia $\quad$ IIb 